\title{
Die Kantenbedingung in der Beugungstheorie elastischer Wellen
}

\author{
Von A.-W. Maue \\ Aus dem Institut für theoretische Physik der Universität Freiburg i. Br. \\ (Z. Naturforschg. 7 a, 387-389 [1952]; eingegangen am 13. Februar 1952)

\begin{abstract}
Es wird untersucht, wie sich eine an einem Materialriß gebeugte elastische Welle in der Nähe der Randkurve der Rißfläche verhält. Hierzu wird der elastostatische Grenzfall unendlich langer Wellenlänge betrachtet.
\end{abstract}

In $n$ der Theorie der Beugung an Schirmen, an deren beiden Oberflächen gewisse Randbedingungen vorgegeben sind, tritt $\mathrm{zu}$ diesen Oberflächenbedingungen und der Ausstrahlungsbedingung im Unendlichen noch eine dritte Bedingung hinzu, die sogenannte Kantenbedingung. Diese stellt vom Standpunkt der allgemeinen Theorie der Randwertprobleme keine selbständige Bedingung dar, ist vielmehr von selbst erfüllt, wenn der Schirm als Grenzfall eines ausgedehnten Körpers mit stetig gekrümmter Oberfläche aufgefaßt wird. Da sich jedoch ein solcher Grenzübergang praktisch schwer durchführen läßt, muß die Kantenbedingung gesondert formuliert werden. Das ist für den Fall der Beugung elektromagnetischer Wellen insbesondere durch Meix ner ${ }^{1}$ geschehen. Im folgenden soll die Kantenbedingung für elastische Wellen diskutiert werden. Den Beugungsschirm soll dabei eine Rißfläche innerhalb des elastischen Materials bilden, deren beide Seiten als freie Oberflächen aufzufassen sind.

Ganz allgemein lassen sich die Verhältnisse in der unmittelbaren Umgebung der Kante übersehen, wenn man den statischen Fall zum Vergleich heranzieht. Beschränkt man sich nämlich auf die Betrachtung eines Bereichs, dessen Ausdehnung klein ist gegen die Wellenlänge, so kann dieWellen- durch die Potentialgleichung ersetzt werden. Auch bedeutet es keine Einschränkung der Allgemeinheit, den Schirm als eben und die Kantenlinie als gerade anzusehen, sofern nur der Bereich klein genug ge-

1 J. Meixner, Z. Naturforschg. 3a, 506 [1948]; Ann. Physik (6) 6, 2 [1949]; W. Braunbek, Ann. Physik (6) 6, 53 [1949]; vgl. auch Bouwkamp, Physica 12, 467 [1946]. Die Kantenbedingung wird wählt wird. Abb. 1 zeigt den Schnitt ( $x$-y-Ebene) senkrecht zur Kante ( $z$-Achse) durch den Rand des Schirms $(y=0, x>0)$.

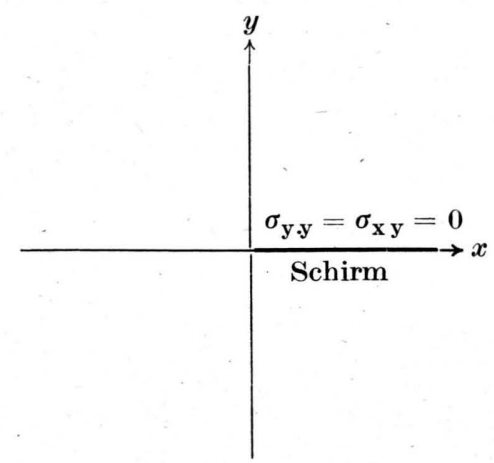

Abb. 1. Koordinaten in der Umgebung einer Stelle der Kante.

Eine skalare Welle $u$ zeigt in der Umgebung der Kante die Abhängigkeit ( $r, \varphi$ Polarkoordinaten):

$u \sim r^{\frac{1}{2}} \sin \varphi / 2 \quad \mid$ bei der Rand- $\mid u=0$ $u \sim$ const. $+r^{\frac{1}{2}} \cos \varphi(2) \quad$ bedingung $\quad\left\{\frac{\partial u}{\partial n}=0\right.$.

Dasselbe gilt für die Komponente $E_{z}$ bzw. $H_{z}$ im elektromagnetischen Falle, wenn der Schirm ideal leitend ist. Für die anderen Komponenten gilt dann

$$
\begin{aligned}
& E_{x}, H_{y} \sim r^{-1 / 2} \sin \varphi / 2, \\
& E_{y}, H_{x} \sim r^{-1 / 2} \cos \varphi / 2 .
\end{aligned}
$$

Entsprechende Formeln lassen sich auch für den allgemeineren Fall einer keilförmigen Kante von

in vielen beugungstheoretischen Arbeiten diskutiert, so u. a. bei P. C. Clemmow, Proc. Roy. Soc. [London], Ser. A 205, 286 [1951], wo sich ausführliche weitere Literaturangaben finden. 
endlichem Öffnungswinkel angeben ${ }^{2}$. Die Forderung des Verhaltens (1), (2) ist für die Welle gleichbedeutend mit der Meixnerschen Forderung der quadratischen Integrierbarkeit (Endlichkeit der Energie).

Einen Überblick über die Verhältnisse im elastischen Falle gewinnt man durch Betrachtung der Airyschen Spannungsfunktion $F$ der zweidimensionalen Elastostatik. Diese genügt der Differentialgleichung

$$
\Delta \Delta F=0
$$

und liefert die Spannungen

$$
\sigma_{x x}=\frac{\partial^{2} F}{\partial y^{2}}, \sigma_{y y}=\frac{\partial^{2} F}{\partial x^{2}}, \sigma_{x y}=-\frac{\partial^{2} F}{\partial x \partial y} .
$$

An einer freien Oberfläche kann gesetzt werden $F=0, \frac{\partial F}{\partial n}=0$, also im vorliegenden Falle

$$
F=0, \frac{\partial F}{\partial \varphi}=0 \text { für } \varphi=0,2 \pi .
$$

Die Lösungen von (3) sind

$$
F=\left\{\begin{array}{l}
r^{ \pm \nu} \\
r^{ \pm \nu+2}
\end{array}\right\} e^{i \nu \varphi}
$$

mit beliebigem $v$, wozu für die $v$-Werte $0, \pm 1$, bei denen die Lösungen (6) teilweise zusammenfallen, noch hinzutreten:

$$
F=\lg r, r^{2} \lg r, r \lg r e^{ \pm i \varphi} .
$$

Aus (6) lassen sich die Lösungen aufbauen, die die Randbedingungen (5) befriedigen (die Funktionen (7) sind hierzu unbrauchbar):

$$
F=r^{1+\frac{n}{2}}\left\{\begin{array}{l}
{\left[\cos \left(1+\frac{n}{2}\right) \varphi-\cos \left(1-\frac{n}{2}\right) \varphi\right]} \\
{\left[\frac{\sin \left(1+\frac{n}{2}\right) \varphi}{1+\frac{n}{2}}-\frac{\sin \left(1-\frac{n}{2}\right) \varphi}{1-\frac{n}{2}}\right]}
\end{array}\right.
$$

mit

$$
n= \pm 1, \pm 2, \ldots .
$$

Der Lösungsschar (8) 'des statischen Problems entspricht eine ebensolche Schar von Lösungen der elastischen Wellengleichung, aus welch letzteren die Lösung des Beugungsproblems aufzubauen ist. Die eindeutige Festlegung dieser Lösung geschieht durch Berücksichtigung 1. der Kantenbedingung und 2. der Randbedingungen im Unendlichen. Daß dabei beiden Bedingungen 1 und 2 gleiches Gewicht

${ }^{2}$ A.-W. Maue, Z. Physik 126, 601 [1949], § 3. zukommt, erkennt man, wenn man das elastische Medium zunächst durch zwei Kreise abgrenzt (Abb. 2 ), deren Radien man dann gegen 0 bzw. $\infty$ gehen läßt. Als Kantenbedingung darf daher gefordert werden, daß genau die Hälfte der Funktionen (8) ausscheidet, d. h. je eines der beiden zu $n= \pm|n|$ gehörigen Lösungspaare, die ersichtlich jeweils

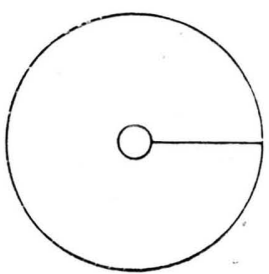

Abb. 2. Begrenztes elastisches Medium.

gleiche $\varphi$-Abhängigkeit aufweisen und sich nur in der $r$-Abhängigkeit unterscheiden. Werden dementsprechend nur positive $n$ beibehalten, so ist das Verhalten der Beugungswelle in Kantennähe durch den niedrigsten $n$-Wert $n=1$ gegeben:

$$
F \sim r^{\frac{3}{2}} \mid \begin{aligned}
& \left(\cos \frac{3 \varphi}{2}-\cos \frac{\varphi}{2}\right) \\
& \mid\left(\frac{2}{3} \sin \frac{3 \varphi}{2}-2 \sin \frac{\varphi}{2}\right) .
\end{aligned}
$$

Hieraus ergeben sich die Spannungen entsprechend (4) nach den Beziehungen

$$
\begin{gathered}
\sigma_{r r}=\left(\frac{1}{r} \frac{\partial}{\partial r}+\frac{1}{r^{2}} \frac{\partial^{2}}{\partial \varphi^{2}}\right) F, \quad \sigma_{\psi \psi}=\frac{\partial^{2} F}{\partial r^{2}}, \\
\sigma_{r \psi}=-\frac{\partial}{\partial r}\left(\frac{1}{r} \frac{\partial F}{\partial \varphi}\right)
\end{gathered}
$$

$\mathrm{zu}$

$$
\begin{aligned}
\sigma_{r r} & \sim-\frac{1}{4} r^{-\frac{1}{2}}\left(3 \cos \frac{3 \varphi}{2}+5 \cos \frac{\varphi}{2}\right), \\
\sigma_{\varphi \psi ;} & \sim \frac{3}{4} r^{-\frac{1}{2}}\left(\cos \frac{3 \varphi}{2}-\cos \frac{\varphi}{2}\right), \\
\sigma_{r \psi} & \sim \frac{1}{4} r^{-\frac{1}{2}}\left(3 \sin \frac{3 \varphi}{2}-\sin \frac{\varphi}{2}\right)
\end{aligned}
$$

und

$$
\begin{aligned}
\sigma_{r r} & \sim-\frac{1}{2} r^{-\frac{1}{2}}\left(\sin \frac{3 \varphi}{2}+5 \sin \frac{\varphi}{2}\right), \\
\sigma_{\varphi \varphi^{\prime}} & \sim \frac{1}{2} r^{-\frac{1}{2}}\left(\sin \frac{3 \varphi}{2}-3 \sin \frac{\varphi}{2}\right), \\
\sigma_{r \psi} & \sim-\frac{1}{2} r^{-\frac{1}{2}}\left(\cos \frac{3 \varphi}{2}-\cos \frac{\varphi}{2}\right) .
\end{aligned}
$$

Hiervon verschwinden, wie es sein muß3, $\sigma_{\varphi \psi}$ und $\sigma_{r \varphi}$ für $\varphi=0,2 \pi$. Durch die Gln. (11) ist die Frage nach demVerhalten der Beugungswelle an der Kante vollständig beantwortet. 
Zur Formulierung der Kantenbedingung ist jedoch diese genaue Kenntnis der Spannungen bei $r=0$ nicht notwendig; sie ist ebensowenig erforderlich wie die Angaben (1) und (2) im skalaren bzw. elektromagnetischen Falle. Es genügt zu wissen, $\operatorname{da} ß$ für den Spannungstensor $\sigma$ gilt

$$
\sigma \sim r^{-\frac{1}{2}} \text { für } r \rightarrow 0,
$$

wofür, da nur ganz- und halbzahlige Potenzen von $r$ überhaupt in Frage kommen, auch geschrieben werden kann

$$
\lim _{r \rightarrow 0} r \cdot \sigma=0 .
$$

Hieraus folgt insbesondere, daß von der Kante keine Kräfte auf das elastische Medium ausgeübt werden, was der Bedingung der freien Oberfläche entspricht, wie sie ja auch an den Schirmflächen gilt. Die Bedingung, daß die von der.Kante insgesamt ausgeübten Kräfte verschwinden, daß also auf einem kleinen Kreise (vgl. Abb. 2) gilt

$$
\begin{aligned}
& \lim _{r \rightarrow 0} r \int_{0}^{2 \pi} \sigma_{r x} \mathrm{~d} \varphi=0 ; \\
& \lim _{r \rightarrow 0} r \int_{0}^{2 \pi} \sigma_{r y} \mathrm{~d} \varphi=0,
\end{aligned}
$$

ist jedoch nicht hinreichend; denn mit (14) wären auch Funktionen (8) mit negativem $n$ verträglich, sofern nur die Integration über $\varphi$ null liefert. (13) besagt vielmehr über (14) hinaus, daß die von der Kante ausgehende Kraft nicht nur insgesamt, sondern nach jeder Richtung hin für sich allein verschwindet, daß also die Integrale (14) auch dann verschwinden müssen, wenn sie über einen ganz beliebigen Winkelbereich $\varphi_{1}<\varphi<\varphi_{2}$ erstreckt wer- den. Es werden damit Kraftwirkungen der Kante von Multipolcharakter ausgeschlossen.

Aus (12) folgt das Verhalten der übrigen kinematischen und dynamischen Größen in Kantennähe, und zwar gilt für die Deformation

$$
\varepsilon \sim \sigma \sim r^{-\frac{1}{2}}
$$

für die Verschiebung

$$
\mathfrak{s} \sim \text { const. }+r^{\frac{1}{2}},
$$

für die kubische Dehnung und lokale Drehung

$$
\operatorname{div} \mathfrak{s} \sim \operatorname{rot} \mathfrak{s} \sim r^{-\frac{1}{2}}
$$

und schließlich für die Energiedichte

$$
W=\frac{1}{2} \sum_{i k} \sigma_{i k} \varepsilon_{i k} \sim r^{-1} .
$$

Auch im elastischen Falle ist demnach die Energiedichte in der Umgebung der Kante integrierbar, was wie im elektromagnetischen Falle zur Formulierung der Kantenbedingung dienen kann, da sich bei Verwendung der Lösung (8) mit $n=-1$ bereits das nicht integrable Verhalten

$$
W \sim r^{-3}
$$

ergeben würde.

Die angestellten, sehr elementaren Überlegungen haben Bedeutung für alle Probleme der Beugung elastischerWellen an Schirmen mit freier Oberfläche (Rissen im Material). Ihre Notwendigkeit ergab sich bei der Behandlung der Beugung elastischer Wellen an der Halbebene ${ }^{3}$.

${ }^{3}$ A.-W. Maue, Z. angew. Math. Mechan. 1952 (im Erscheinen). 